EESTI NSV TEADUSTE AKADEEMIA TOIMETISED. XI KÖIDE POOSIKALIS-MATEMAATILISTE JA TEHNILISTE TEADUSTE SEERIA. 1962, NR. 4

ИЗВЕСТИЯ АКАДЕМИИ НАУК ЭСТОНСКОЙ ССР. ТОМ ХІ СЕРИЯ ФИЗИКО-МАТЕМАТИЧЕСКИХ И ТЕХНИЧЕСКИХ НАУК, 1962, № 4

\title{
УСКОРЕНИЕ И ЭНЕРГЕТИКА НАРАСТАНИЯ МАГНИТНОГО ПОТОКА
}

\author{
B. CAPB,
}

кандидат технических наук

Существование переходных процессов в природе вызвано тем, что преобразование энергии из одного вида в другой происходит всегда с какой-то ограниченной мощностью и поэтому связано с затратой времени.

Вследствие тесной связи между электрическими и магнитными явлениями в электромагнитных переходных процессах нарастание энергии магнитного поля происходит за счет потребляемой электрической энергии, а убывание магнитного поля всегда связано с генерированием электрической энергии.

Скорость нарастания магнитного потока при включении заданной электромагнитной нагрузки (например, обмотки возбуждения или управления) определяется:

а) развиваемой источником питания мощностью,

б) коэффициентом полезного действия (к.п.д.) процесса преобразования потребляемой электрической энергии в энергию магнитного поля.

Скорост!, убывания магнитного поля определяется скоростью преобразования энергии магнитного поля в какой-либо другой вид энергли. В сушествуюших схемах управления электромагнитными устройствами энергия убывающего магнитного поля преобразуется в основном в тепло, что практически исключает возможность дальнейшего использования этой энергии в последующих электромагнитных процессах.

До настоящего времени описано большое количество методов и конкретных схем для ускорения электромагнитных переходных процессов, в частности для ускорения нарастания магнитного потока. Переходный процес определен преобразованием различных видов энергии по времени Поэтому для систематизации существующих способов ускорения электромагнитных переходных процессов и в особенности для выяснения возможных новых методов ускорения следует считать целесообразным рассмотрение энергетики электромагнитных переходных процессов.

В статье рассматривается связь между ускорением и энергетнкой электромагнитных переходных процессов при включении активно-индуктивной нагрузки на постоянное напряжени'е.

По итогам рассмотрения энергетики переходных процессов предлагаются также некоторые новые принципы дальнейшего увеличения скорости нарастани'я магнитного потока. 


\section{Включение линейной активно-индуктивной нагрузки на постоянное напряжение}

Рассмотрим энергетическое протекание переходного процесса при включении схемы рис. 1. Для большей наглядности применяем относительные величины, принимая за единицу напряжение питания $U$, уста-

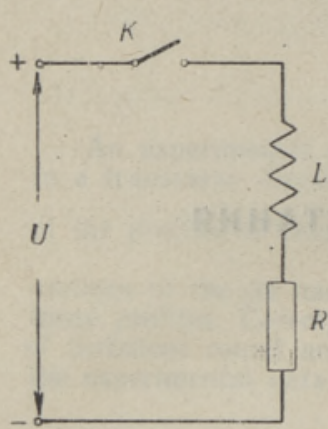
новившийся ток $I=U: R$ и промежуток времени, равный постоянной времени цепи, $T=L: R$. В этом случае единицами являются также индуктивность $L$, активное сопротивление $R$ и мощность $P$, развиваемая источником питания в установившемся режиме. Строчными буквами обозначаем относительные значения мощностей $(p)$, тока $(i)$, падения напряжения $(u)$ и времени $(t)$.

Рис. 1. Рассматриваемая цепь с активно-индуктивной нагрузкой.

С учетом вышеуказанного, переходный процесс при замыкании выключателя $K$ описывается следующими формулами:

TOK

$$
i=I\left(1-e^{-\frac{t}{T}}\right)=1-e^{-t}
$$

активное падение напряжения

$$
u_{R}=R i=1-e^{-t}
$$

индуктивное падение напряжения

$$
u_{L}=L \frac{d i}{d t}=e^{-t}
$$

мощность, развиваемая источником питания,

$$
p=U i=1-e^{-t}
$$

мощность тепловых потерь

$$
p_{R}=u_{R} i=\left(1-e^{-t}\right)^{2}
$$

полезная мощность, соответствующая той части электрической энергии, которая преобразуется в энергию магнитного поля,

$$
p_{L}=u_{L} i=e^{-t}-e^{-2 t} \text {; }
$$

мгновенный к.п.д. преобразовательного процесса

$$
\eta=\frac{p_{L}}{p}=\frac{u_{L}}{U}=e^{-t}
$$


энергетический к.п.д.

$$
\eta_{9 \mathrm{H}}=\frac{\int_{0}^{t} p_{L} d t}{\int_{0}^{t} p d t}=\frac{0,5\left(1-e^{-t}\right)^{2}}{t+e^{-t}-1} .
$$

Характеристики протекания переходного процесса в относительных единицах изображены на рис. 2 , из которого наглядно видны основные недостатки естественного, т. е. до конца экспоненциального, переходного процесса, которые способствуют увеличению длительности нарастания магнит ного потока:

\section{Рис. 2.}

Протекание переходного процесса при включении активно-индуктивной нагрузки на постоянное напряжение.

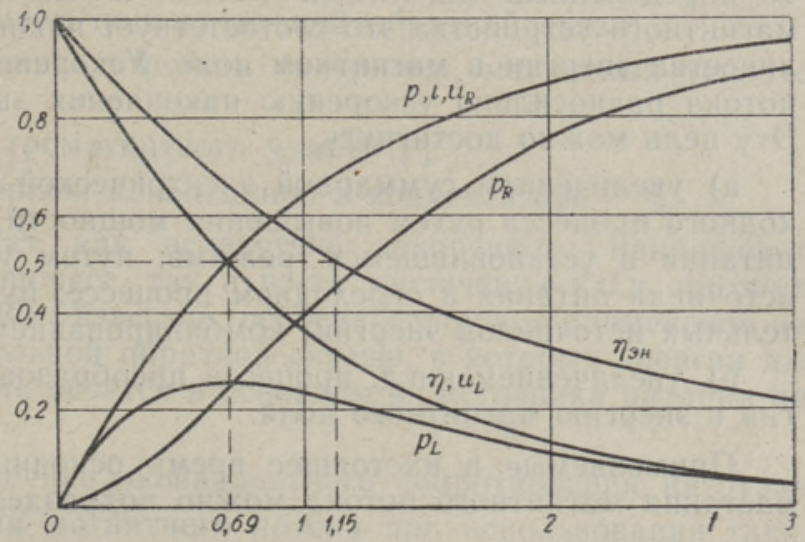

а) мощность, развиваемая источни'ком управления в начале переходного процесса, значительно меньше его номинальной мощности;

б) к.п.д. процесса преобразования электрической энергии в энергию магнитного поля высок только.в начале нарастани'я магнитного потока.

Әкспоненциальный переходный процесс считается практическц оконченным при $t=3$ и, соответственно, $i=0,95$. В этот момент энергия, накопленная в магнитном поле,

$$
W_{L}^{\prime}=\int_{0}^{3} p_{L} d t=0,45
$$

а энергия, израсходованная источником питания,

$$
W^{\prime}=\int_{0}^{3} p d t=2,05
$$

Для сравнения напомним, что при выбранных единицах энергия магнитного поля при установившемся токе $I=1$ равняется

$$
\frac{L / 2}{2}=0,5 \text {. }
$$

Соответственно при $t=3$ энергетический к.п.д.

$$
\eta_{9 н}^{\prime}=\frac{W_{L}^{\prime}}{W^{\prime}}=0,219
$$


Таким образом, при нефорсированном, до конца экспоненциальном переходном процессе только примерно $\frac{1}{5}$ из потребляемой электрической энергии преобразуется в энергию магнитного поля, остальная же часть преобразуется в тепло.

\section{Существующие способы ускорения нарастания магнитного потока}

В конце переходного процесса в сердечнике устанавливается какойто определенный магнитный поток. В случае конкретного электромагнитного устройства это соответствует накоплению определенного количества энергии в магнитном поле. Ускорение нарастания магнитного потока равносильно ускорению накопления энергии в магнитном поле. Эту цель можно достигнуть

а) увеличением суммарной электрической мощности во время переходного процесса путем повышения мощности, развиваемой источником питания в установившемся режиме; путем улучшения использования источника питания в переходном процессе; путем применения дополнительных источников энергии; комбинированием перечисленных методов;

б) увеличением к.п.д. процесса преобразования электрической энергий в энергию магнитного поля.

Применяемые в настоящее время основные методы ускорения нарастания магнитного потока можно подразделить на четыре группы.

1. Постоянное увеличение напряжения источника питания с одновременным увеличением сопротивления включаемой цепи.

В таких схемах с постоянным напряжени'ем питания ускорение переходного процесса происходит за счет увеличения расхода энергии и соответственно установившейся мощности источника питания, но в случае применения нелинейных добавочных сопротивлений, полупроводниковых триодов $\left[^{1}\right]$ и при' шунтировании добавочного сопротивления во время переходного процесса ускорение происходит также вследствие улучшения к.п.д. преобразовательного процесса. Хотя такие схемы очень просты, их недостатком является увеличение тепловых потерь, увеличение мощности и габаритов источника питания, а также уменьшение коэффициента усиления управляемого устройства.

Когда нагрузку питают через выпрямитель от источника переменного напряжения, то добавочное сопротивление может быть включено в участок переменного тока и соответственно являться реактивным. В этом случае не происходит существенного увеличения тепловых потерь. В литературе рассмотрены схемы с индуктивным добавочным сопротивлением $\left[{ }^{2}\right]$. Наряду с этим также возможно при'менение емкостного добавочного сопротивления, так как независимо от характера реактивного сопротивления падение напряжения на реакти'вном сопротивлении возникает и растет вместе с увеличением тока во включаемой цепи.

2. Периодическое и'ли однократное увеличение напряжения питания на ограниченный промежуток времени без увеличения активного сопротивления включаемой цепи.

Сюда относятся схемы с и'мпульсным питанием [3] и схемы форсирования путем повышения напряжения на время переходного процесса.

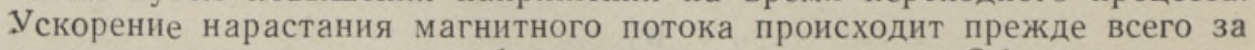
счет увеличения к.п.д. преобразовательного процесса. Объясняется это 
тем, что энергия, соответствующая нужной величине магнитного потока, накапливается в магнитном поле уже в первой стадии экспоненциального переходного процесса, когда к.п.д. высок. Эти схемы с энергетической точки зрения экономичны и поэтому находят широкое применение, например, в автоматизированном электроприводе. Но в большинстве случаев их применение связано с увеличением габаритов источника питания, так как габариты электромагнитных устройств заданной мощности зависят также от относительной величины максимального напряжения.

3. Применение наряду с источником управления еще дополнительных источников энергии.

Этот метод реализуется различными типами схем:

а) схемы с применением положительной обратной связи;

б) каскадное включение устройств (например; магнитных усилителей);

в) применение «электромагнитного удара» $\left[{ }^{4}\right]$;

г) использование энергии, накопленной в конденсаторе $\left[{ }^{5,6}\right]$.

Ускорение происходит как вследствие ускоренного направления энергии в переходный процесс, так и из-за увеличения к.п.д. преобразовательного процесса. Как известно, особенно широкое применение находят схемы с положительной обратной связью, в которых энергия для создания магнитного поля берется в основном и'з источника питания нагрузочной цепи.

4. Временное уменьшение эквивалентного сопротивления нагрузки.

Ускорение нарастания магнитного потока при использовании таких схем связано с увеличением индуктивного падения напряжения на один виток. Это вызывает увеличение к.п.д. преобразовательного процесса и развиваемой источником питания мощности. Принципиально метод временного уменьшения эквивалентного сопротивления является прогрессивным, так как позволяет лучше использовать источник питания в переходном процессе и зачастую допускает ускорение переходного процесса без соответствующего увеличения номинальной мощности и габаритов источника питания. Однако недостатком применяемой схемы $\left[{ }^{7}\right]$ для реализации этого метода путем временного шунтировани'я одной части витков является появление короткозамкнутого контура, который увеличивает расход энергии в переходном процессе. Этот недостаток становится особенно заметным при частом срабатывании электромагнитного устройства.

Мы классифицировали возможные способы ускорения нарастания магнитного потока в случае управления уже заданным электромагнитным устройством. Но если заданным является только выходная мощность или какое-либо другое выходное воздействие, то ускорению пере ходного процесса может способствовать еще применение качественных материалов, выбор оптимальной конструкции и увеличение частоты питания нагрузочной цепи. С энергетической точки зрения эти мероприятия увеличивают отношение выходной мощности к энергии', накопленной в электромагнитном устройстве. 


\section{Некоторые возможности дальнейшего увеличения скорости нарастания магнитного потока}

Переходный процесс почти всегда можно ускорить до желаемого предела путем увеличения расхода энергии при создании управляющего магнитного поля. Но это связано с увеличением габаритов источников управления, уменьшением коэффициента усиления управляемого устройства, увеличением тепловых потерь или потребностью в дополнительных источниках питания. Кроме того, зачастую мощность источника управления ограничена.

Поэтому целесообразно достигнуть требуемое быстродействие электромагнитного устройства прежде всего

а) созданием оптимальной конструкции, обеспечивающей нужное выходное воздействие при наименьшей энергии управляющего магнитного поля;

б) выбором таких схем форсирования, которые основаны на улучшении к.п.д. процесса преобразования, а также на более совершенном использовании источника управления, но не требуют существенного повышения установившейся мощности и габаритов источника управления.

Считаем, что электромагнитное устройство уже задано и ограничиваемся случаями, когда управляющее магнитное поле создается в основном за счет энергии источника управления.

Если можно устранить возникновение короткозамкнутого контура, то при непериодических включениях одним из наиболее целесообразных следует считать ускорение временным уменьшением эквивалентного сопротивления нагрузки. Это возможно путем применения в существующей схеме [7] дополнительного вентиля В (рис. 3). Вентиль В следует выбрать, исходя из номинального тока обмотки и максимального обратного напряжения

$$
u_{\mathrm{OM}} \lesssim U \frac{w 2}{w 1} \text {. }
$$

Нормально замкнутый контакт РТ открывается при достижении магнитным потоком заданной величины.

Некоторым недостатком простейшей схемы на рис. 3 является то, что во время переходного процесса в создании магнитодвижущей силы участвует только обмотка w1. Вследствие этого для достижения номинальной величины магнитного потока ток в обмотке 1 должен превышать номинальный ток управления в $\frac{w 1+w 2}{w 1}$ раз. В большинстве случаев как источники питани'я, так и обмотки управления допускают кратковременную токовую перегрузку, что и является большим преимуществом схем форсирования, используюших временное уменьшение эквивалентного сопротивления нагрузки.

Но можно построить схему форсирования и таким образом, чтобы во время пєреходного процесса в работе участвовали все части обмотки. Это уменьшило бы активное падение напряжения, а вместе с тем и тепловые потери во время переходного процесса. Для этого обмотка электромагнитного устройства разделяется на две или, в общем случае, на большее число равных частей -w1, w2, ..wn (рис. 4), соединяемых последовательно через вентили $B_{1}, B_{2}, \ldots B_{n-1}$. В начале переходного процесса через нормально замкнутые контакты (например, реле тока РТ) части обмотки $w 1, w 2, \ldots$. wn соединены параллельно источнику 
питания. Вследствие этого коэффициент форсировки $\boldsymbol{\alpha}=n$. Реле РТ срабатывает при токе, равном установившемуся току обмотки после размыкания нормально замкнутых контактов. Тем самым переходный процесс заканчивается. Как известно, уже при коэффициенте форсировки $\alpha=2$, что соответствует разделению обмотки на две равные части, достигается ускорение процесса нарастания магнитного потока примерно в $5-7$ раз.

Когда управляющим устройством является реле или контактор, то вместо контактов дополнительного реле РТ можно использовать нормально замкнутые контакты управляемого устройства.

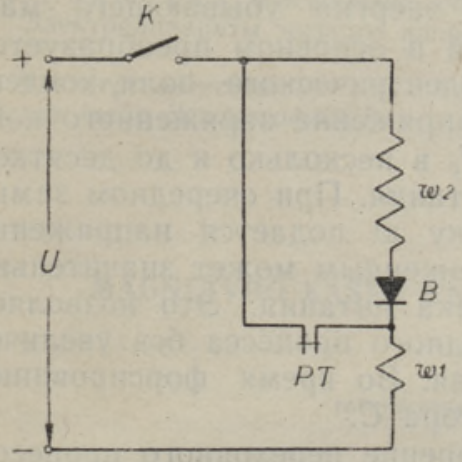

Рис. 3. Схема с вентилем В для устранения короткозамкнутого контура.

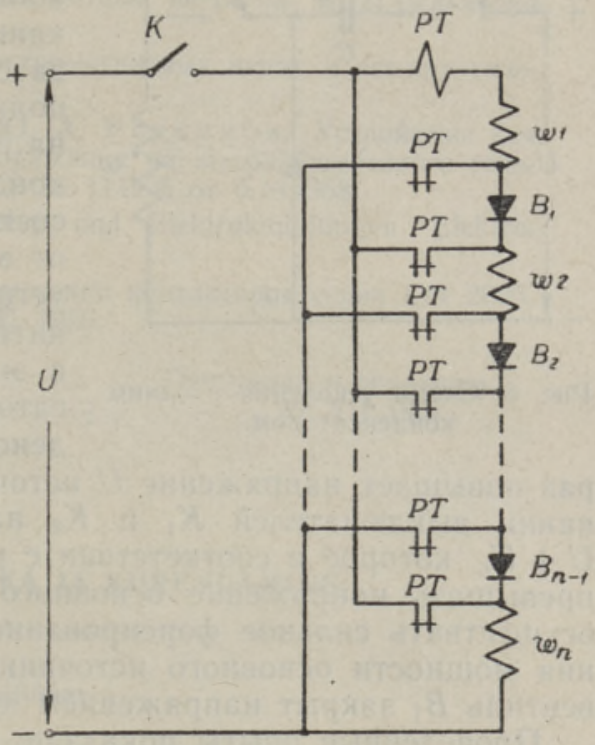

Рис. 4. Принципиальная схема форсирования с уменьшением эквивалентного сопротивления нагрузки в $n^{2}$ раз.

Можкно получить бесконтактную схему форсировки путем замены нормально замкнутых контактов бесконтактными коммутирующими устройствами или конденсаторами соответствующей величины. Схема с ускоряющими конденсаторами при $n=2$ изображена на рис. 5 .

Электромагнитные устройства часто работают в режиме повторных включений и выключений. При включении электроэнергия, необходимая для создания магнитного. поля, берется из источника управления, причем часто ограниченная мощность управления ограничивает скорость нарастания магнитного потока. Несмотря на это, при выключении энергия убывающего магнитного поля обыкновенно преврашается просто в тепло. Как известно, ускорение нарастания магнитного потока сверх предела, определенного мощностью источника питания, возможно, если для созлания необходимого магнитного поля огновременно с энергией основного источника управления использовать еше

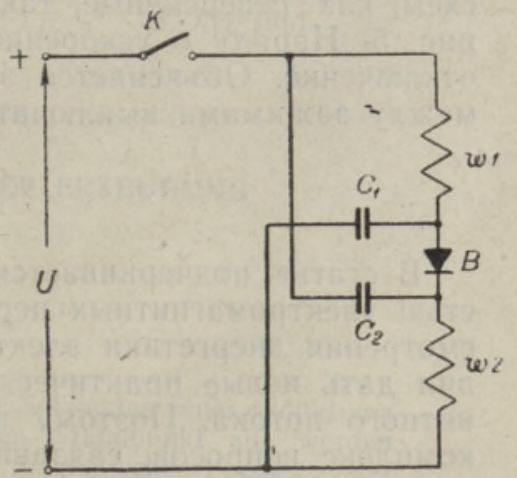

Рис. 5. Схема ускорения с двумя конденсаторами

дополнительную энергию. Для получения такой дополнительной энергии предлагаем преобразовать энергию убывающего магнитного поля не в 
тепло, а в какой-нибудь другой вид энергии, допускающий накопление и повторное использование накопленной энергии. Наиболее простым и довольно удобным является накопление энергии в электрическом поле

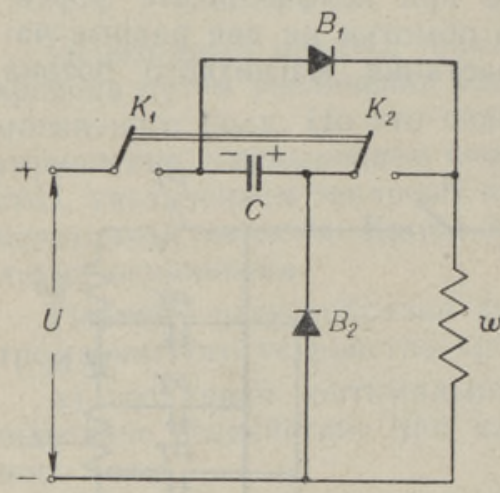

Рис. 6. Схема ускорения с одним конденсатором. конденсатора. На рис. 6 приведена одна из возможных схем. При установившемся режйме обмотка управления ш питается через выключатель $K_{1}$ и вентиль $B_{1}$; конденсатор $C$ разряжен. После размыкания выключателей $K_{1}$ и $K_{2}$ конденсатор за счет энергии убывающего магнитногі поля заряжается полярностью, указанной на рис. 6. При соответственно выбранном конденсаторе (вес которого обыкновенно составляет только несколько процентов от веса управляемого электромагнитного устройства) энергия убывающего магнитного поля в основном преобразуется в энергию электрического поля конден- сатора, а напряжение заряженного конденсатора $U_{c}$ в несколько и до десятков раз повышает напряжение $U$ источника питания. При очередном замыкании выключателей $K_{1}$ и $K_{2}$ на обмотку ш подается напряжение $U+U_{c}$, которое в соответствии с вышеизложенным может значительно превышать напряжение основного источника питания. Это позволяет осуществить сильное форсирование переходного процесса без увеличения мошности основного источника питания. Во время форсирования вентиль $B_{1}$ закрыт напряжением конденсатора $C$.

Проведеннье опыты показали, что ускорение переходного процесса в десять и больше раз можно получить сравнительно легко. При этом из принципа работы схемы вытекает (что также подтверждается экспериментальной проверкой), что применение накопляющих и ускоряющих конденсаторов необходимо сочетать с предотвращением существенных вихревых токов в магнитопроводе.

Вышеописанная схема не является единственно возможной для реапизации метода накопления и повторного использования энергии убывающего магнитного поля. Возможны различные другие разновидности схем, как реверсивные, так и нереверсивные, в том числе и схема на рис. 5. Наряду с ускорением такие схемы обеспечивают безыскровое отключение. Объясняется это отсутствием существенного напряжения между зажимами выключателя в первый момент после выключения.

\section{Заключение}

В статье подчеркивается тесная связь между энергетикой и скоростью электромагнитных переходных процессов. Уже первый этап рассмотрения энергетики электромагнитных переходных процессов позволил дать новые практические методы для ускорени'я нарастания магнитного потока. Поэтому необходимо всесторонне рассмотреть весь комплекс вопросов, связанных с энергетикой электромагнитных переходных процессов. Особенно существенные практические результаты должно дать рациональное решение проблемы накопления и повторного использования энергии убывающего магнитного поля в устройствах, работающих в режиме повторного нарастания и убывания магнитного поля. Сюда относятся и устройства, управляемые реверсированием магнитного потока. 


\title{
ЛИТЕРАТУРА
}

1. М. Н. Фесенко, Об одном методе форсирования переходного процесса, Электричество, № 6, 1960.

2. В. Е. Боголю 6 ов, В. Л. Д ятлов, Снижение постоянной времени цепи управлення электропривода, содержащего выпрямительный мост, Тр. Моск. энерг. ин-та, вып. 22, 1956.

3. В. А. Олей н и ов, Работа магнитного усилителя в импульсной системе регулирования и управления. Изв. Ленингр. электротехн. ин-та им. В. И. Ульянова (Ленина), вып. XXXIV, 1958.

4. А. Д. Р а б и н о в и ч, Явление «удара» в электромагнитных цепях и его практическюе применение, дисс. М., 1955.

5. Б. М. Шля пошников, А. Я. Рубин, Ю. А. Резников, Устройство для форсирования процесса установления напряжения на выходе шунтового генератора постоянного тока, авт. свид. СССР № 114905 от 6.10.1958.

6. R. Weber, Stosserregung von Elektromagneten und Elektrokupplungen. ElektroAnzeiger, Nr. 3-4, 1960.

7. Л. А. Р одште й н, Расширение области применения контакторов серии ҚМ 2000, Электроаппараты низкого напряжения, М., 1959.

Ннститут энергетики

Академии наук Әстонской ССР
Поступила в редакцию

25. XII 1961

\section{MAGNETVOO KASVU ENERGEETIKA JA KIIRENDAMINE}

\author{
V. Sarv, \\ tehnikateaduste kandidaat
}

Resümee

Artiklis analüüsitakse siirdeprotsessi energeetikat induktiivse aktiivkoormuse lülitamisel alalispingele. Lähtudes energeetilisest käsitlusest, liigitatakse tänapäeval kasutatavad magnetvoo kasvu kiirendamise meetodid ning esitatakse uusi otstarbekaid võimalusi magnetvoo kasvu kiirendamiseks.

Artikli eesmärgiks on rōhutada elektromagnetiliste siirdeprotsesside energeetika ja nende kulgemise kiiruse tihedat seost ning pōhjendada vastavate uurimistööde süvendamise ning laiendamise vajadust.
Eesti NSV Teaduste Akadeemia
Energeetika Instituut
Saabus toimetusse
25. XII 1961

\section{DIE BESCHLEUNIGUNG UND ENERGETIK DER ERZEUGUNG MAGNETISCHEN FLUSSES}

\author{
V. Sarv \\ Zusammenfassung
}

Im Artikel wird die Energetik des Schaltvorganges beim Schliessen einer induktiverr Wirklast auf Gleichspannung analysiert. Vom energetischen Standpunkt aus werden die heutigen Methoden für die Beschleunigung der Erzeugung magnetischen Flusses systematisiert und einige neue Beschleunigungsmassnahmen vorgeschlagen.

Aufgabe des Artikels ist, den engen Zusammenhang zwischen Energetik und Geschwindigkeit der elektromagnetischen Schaltvorgänge zu betonen.

\section{Institut für Energetik}

der Akademie der Wissenschaften der Estnischen SSR
Eingegangen am 25. Dez. 1961 